\title{
PHENOMENA OF FUNCTIONAL DIFFERENTIATION AND FRACTAL FUNCTIONALITY
}

\author{
IRINA TROFIMOVA \\ Department of Psychiatry and Behavioral Neuroscience, McMaster University, Hamilton, Canada.
}

\begin{abstract}
The phenomena of vertical and horizontal emergence are analysed in terms of functional differentiation (FD), the concept of fractal functionality (FF), the concept of the zone of proximate development and an application to iterative map techniques. These theoretical components are used to trace an evolution of structures at various levels of organization and to derive several universal evolutionary principles. The implications of these principles are: (1) "building blocks" of natural systems are performed uniquely and only once, they emerge, change and disappear, and therefore cannot be considered as Lego-like "bricks" for these systems; (2) "building blocks" develop not prior to, but simultaneously with the emergence of a macro-system, to which they are associated, and may continue to change even after the macro-system is established; (3) the existence of functional groups "on the diagonal" affects the subdivisions of horizontal distributions; (4) soft associations of elements to functional systems of several levels of complexity speaks against a vertical division of complexity levels and against a horizontal division of building blocks.

Keywords: diagonal evolution, emergence, functional differentiation FD-3 operators, functionality, neurotransmitters, zone of proximate development.
\end{abstract}

\section{EMERGENT NATURE OF LIVING AND SOCIAL SYSTEMS: MOVING AWAY FROM LEGO SCIENCE}

The concept of emergence is fundamental to complex systems research, yet its significance is still underestimated in the majority of the sciences. Most natural sciences describe their objects using the "Lego" approach, i.e. as being built up from a collection of smaller, constant fundamental parts. This follows the reductionist philosophy that presumes that the behaviour of natural systems can be derived from the properties of their fundamental components, i.e. from knowing "what kind of Lego blocks this stuff is made of". Scientific analysis describes the interactions between these components mostly in mechanical terms. Thus, physiology is focused on the actions of organ systems within bodies; biology is focused on biomolecules and their interactions, especially those forming genes; physicists are focused on elementary particles and the search for grand theories of everything based on their properties.

Remarkably, the emergent and transient nature of life and social systems has been recognized for several millennia even while ignored by Western science [1-4]. There is abundant evidence that natural systems and phenomena, no matter how large or structurally stable they appear, have limited life spans and throughout their existence continue to change both structurally and functionally and then die. This is readily apparent in the life and social sciences where open, adaptive, developing systems with fungible components are the norms. This is true in physics and chemistry as well although such systems have tended to be ignored for methodological and philosophical reasons. Unfortunately, this has led to the development of mathematical models and methods ideally suited 
to reductionist, "Lego" science, and ill suited to the study of natural systems. The wholesale adoption of these methods by biology, economics, social science and psychology, has led to the widespread misapplication of linear methodologies such as factor analysis, leading to stalled progress, misleading and even erroneous results $[5,6]$

For example, in evolution theory, many models use a concept of traits/characteristics $z_{i}$ that are either inherited or not under the process of natural selection, using a fitness function $w_{i \text {. }}$, and discuss the covariance of these traits at the individual, group or population levels. Problems arise, however, when we attempt to partition the evolving systems and their traits into the units of analysis. After all, these systems are not isolated but rather are strongly contingent on multiple systems at various levels of a conditional hierarchy of organization. As Cairns-Smith put it, "quite simply, in central biochemistry everything depends on everything" [7] (p. 94). Evolution theories focus primarily on structurally observable properties of biological species, such as phenotype or genotype, but rarely provide across the board comparisons of more fluid features associated with the emergence and maintenance of these species. The partitioning of traits/characteristics of species is also biased by theoretical traditions, often under-estimating characteristics related to within-species, between-species and species-environment interactions. More importantly, these systems are transient, and relying on their presence or that of their traits might lead to wrong results in computer modelling of evolutionary processes. The emergence, divergence and disappearance of biological species through genetic mutations were often attributed to a mechanical re-assembly of previously established genetic structures, while the dynamic nature of DNA and background processes were often not considered important until the mutation took place. However, it is mainly these processes that show up as a mutation.

The concept of emergence is close to the concept of evolution: initially, it was thought that evolved structures depended on their predecessors entirely whereas emergence related to something lacking predecessors. Nowadays evolution theory considers evolution as based on multiple parallel, independent processes that are capable of bringing new emergent features in a sudden fashion [8]. I contend that the concepts of evolution and emergence can be equally applied to all natural phenomena, even very transient phenomena. Indeed evolution may be considered as a special case of emergence.

\section{ADDRESSING TRANSIENCE: PERFORMANCES (PRF-S) AS UNITS OF ANALYSIS}

Considering the transience of living systems, we have to wonder what holds these systems together as consistent objects thus making them observable. The existence of natural systems is described here as a composition, construction or "performance" via synergetic interactions between multiple dynamical sources. The concept of "performance" (denoted by "prf") is introduced here for the formal description of a natural system, analogous to features usually attributed to the performance of a play. These features include:

Process-like nature: the performance of a play is a process, a sequence of multiple overlapping events unfolding within a certain time frame. Similarly, natural systems, whether social, biological or physical, are the result of multiple events (or processes, that are also events but spread over time) contributing to their existence. Natural systems also manifest for finite durations and may exhibit a discontinuous existence (emerging several times with some periods of non-existence in-between).

Existent does not mean observed: there is a swarm of components that contribute to a given perf, and the most prominent perf is merely an approximation of the most consistent elements and only those chosen by an observer. For example, the lead up to a play's performance involves multiple rehearsals and many failures - nevertheless these form part of an essential swarm of events required to ensure that the final product is of quality, and so should be considered as part of this perf. Only 
the final performance is likely to be acknowledged by the audience (observer). Likewise, forest mushrooms have an extensive underground fungal bed providing many semi-ready buds, most of which won't survive to the observation stage. This background network of prfs provides a storage of drafts that new prfs might use as building blocks.

Low rates of being observed: many prfs, even in a semi-ready state, are never observed because their components are not consistent enough to capture the attention of an observer. Some consistent components contributing to prfs ("service components") may still be invisible because only the "artists" have observers who make them visible (anecdotally the ratio of actors to stage crew is roughly 1:10). Thus transience in natural systems may be under-recognized because there is simply a lack of appropriate observers to render the associated prfs visible.

Local utility of consumer/observation bias: Results of an observation of a natural system depend on the nature of the observer. An ant perceives a chair differently than a human. Those aspects of a natural system that are observed depend upon the presence of an observer that reacts to specific consistencies within the swarm of prfs that is the system. Many plays are never observed because the consumer "is not ready to buy it". By analogy, the concept of fitness in evolution theory describes a correspondence between traits, or characteristics of biological prfs and environmental biases towards or against these traits. Whereas globally, evolution and emergence are likely neutral, locally, consistency in the emergence of structures suggests a "lobby" from contributing factors that capitalizes on the properties of these structures. In this sense, we can talk about temporal and local fitness. Vrba and Gould [9] underlined that the natural selection of species encompasses those heritable character-environment interactions that cause differences in rates of birth or death among varying individuals (high fitness), but this doesn't exclude the emergence of less fit characters. Different species use different sensory spectra to examine their environments and generally do not react to the properties of objects that cannot be perceived, thus creating an observation bias. When biologists reason about features of species they might also suffer an observation bias steaming from specific, socio-cultural properties of human nature. For example, it has been noticed that humans of different sex and temperament perceive neutral and abstract concepts differently [4, 6, 10, 11]. In general, the selectivity of observation means, by definition, that most properties are not being perceived. Returning to the play analogy, rehearsals, advertising and administration of a performance are essential to its existence, however, they are not registered by the audience members who only came to see the show. They would appear to be low fitness prfs to the audience yet are vitally important to the performance of the play. Utility is thus local and subject to observer bias.

Global neutrality of emergence: the emergence of features of biological species often has little to do with a better adaptation to their environment, even though those features that facilitate adaptation promote survival. Instead of viewing the properties of a species as always arising from increased fitness and adaptation, several evolution theories pointed to the rather neutral character of evolution [12-15]. This brings the concept of evolution closer to the concept of emergence. Returning to the play metaphor, "consumers", representing the environmental reinforcement of certain traits, are often unresponsive to the majority of a prf's properties and focus only on those aspects that benefit themselves, however, this doesn't mean that "overlooked" traits do not survive in the production if they do not cause harm to a prf's existence.

Mutual agreement principle in multiple choices: it is likely that natural systems, or perfs, are not actively seeking survival, contrary to common belief. It is just that those who have better compatibility with favouring factors (including cooperation and employment strategies) have better chances to survive longer [16]. Systems enter into interactions, regardless of whether the search for required prfs is active or passive, based upon comparisons of their compatibility to one another, in terms of salient features governing their participation in a joint performance. Similar to speed dating or to 
employee recruitment, the greater the compatibility, the longer the relationship will be maintained, and it will be longest if all prfs performing in an ensemble "agree" to it [18]. This "mutual-agreement" sorting is happening across all levels of complexity and time frames, and thus vertically, horizontally and diagonally.

\section{COEVOLUTION AT MULTIPLE LEVELS: IF EVOLUTION GOES DIAGONALLY...}

Two distinct forms of emergence are described in the complex systems literature: vertical and horizontal emergence.

"Vertical emergence" is described as a process of composition, a grouping of lower-level "bricks" into some superordinate-system. Fundamental particles form atoms which form molecules, organelles, cells, organs, organisms, families, societies, with causation acting from the bottom up and from the top down (Andersen et al. 2000). Hierarchical taxonomies of natural systems are complemented by descriptions of how upper levels of organization regulate the bottom processes, or how the nature of "brick" elements of super-systems determines the functioning of super systems.

"Horizontal" emergence occurs among systems existing within the same phenomenological level. The examples of horizontal emergence are predator-prey and host-parasite dynamics, symbiosis, sex and kin selection [17], collective mode phenomena [18-20] including collective intelligence [21], functional differentiation between cells or organisms [22], and an impact of a spectrum of sociabilities of agents on behaviour of a population [23]. Since Darwin it had been noticed that living systems often co-evolved resulting in correlated changes among their features. Co-evolution was mostly described as applying to systems at the same level of organization (e.g. co-evolution of organisms [23], or even co-evolution of galaxies [24], but not across the levels.

This paper proposes the idea that in evolution emergence may go diagonally. Diagonal evolution, or dievolution is the view that observable and structured natural systems developed in evolution "on a diagonal", with several levels of natural selection working simultaneously and changing the structures at these levels accordingly. Suppose that a natural system of interest is said to exist at level $\mathrm{i}$. Then component (lower) levels are denoted as $i-1, i-2, i-3$ etc while organizational (higher) levels are denoted $i+1, i+2, i+3$ etc. The dievolution concept suggests that specific features of the structures at the level $i$ are the results of natural selection occurring reciprocally at a minimum of three levels. Here are the six important features of dievolution that are often overlooked in the literature on evolution and emergence:

1. The dievolution process requires the contribution of (at least) three levels of organization. In other words, a structure at level $i$ cannot emerge unless there are processes at the $i+1$ level that consistently select for the properties of the $i$-level structure, and unless there is mutual agreement (correspondence) between the $i+1$ and $i-1$ levels. Consequently, changes at either the $i+1$ or $i-1$ levels can compromise the compatibility between them, without which the resulting $i$-level structure will start to deteriorate and disassemble. Thus, the factors underlying the mutation or disappearance of biological species are not always observable and always should be analysed in terms of an interaction between lower and upper levels of organization, and not just lower (e.g. accidental gene mutation) or upper (e.g. climate change) levels only. A similar idea about multi-level selection was independently expressed in the Multilevel Selection Theory, MLS [20, 26] that also suggested that higher-level selection can be a significant evolutionary force for lower-level selection. The difference between MLS and dievolution theory is the more strict statement about simultaneous multi-level evolution for at least three levels, with the middle one produced through the interaction with the other two, the lower one becoming more specialized and partitioned while the top is sketched up and starts developing almost simultaneously with the middle one. 
2. An increase in the consistency of structures emerging at the $i$ level affects, in turn, selection processes at the $i-1$ level and creates a dispositional engagement with processes at the $i+2$ level. Securing emergence at $i$ level starts a process of setting up the $i+2$ level of organization. This type of dynamics was noticed in developmental psychology by Vigotsky and informed his concept of a Zone of Proximate Development (ZPD). ZPD is observed when children are too young to produce a certain behaviour but, when exposed to suitable degrees of freedom in actions, they choose a ZPD that suits their motivations and needs and tune the development of their skills towards these certain activities. In neuropsychology, it has been noticed that in the development of an action, the choice of neuronal ensembles is determined by the overall task that this action should contribute to, and such neuronal and behavioural integration proceeds with constant feedback adjusting the ensembles and the content of the task itself. In line with Gould's idea [8], the evolution (not development) of natural systems might not come as the gradual growth of a state space; instead it maps distant future limits ahead of time and fills out the intermediate states in a non-sequential manner (Fig. 1).

3. Structures possessing a diagonal subordination have more chances to maintain their consistency in step-by-step emergence than structures with either vertical or horizontal subordination alone. Diagonal subordination is a contribution of factors from several levels to prf's production in an overlapping manner: distinct prf-s can be produced out of the same components and selecting them levels, and contributing selecting factors act, of course on different prfs. Analogous to a stage crew having part-time jobs in several theatres, the same components of natural systems can be used in different perfs and even provide different functions. Plus there is a multiplicity of time frames in prf-s components: the temporal features (timing of onset, offset, duration) and of the factors contributing to their emergence are often only weakly correlated. By analogy with holography, the more perspectives converge on the same point, the sharper the image, and even in performing a play there are benefits for having several alternatives for leading artists, advertising campaigns, stage crews and so on. Likewise in neurophysiology, it has been found that the same action can be regulated by different neuronal ensembles even though there is some localization

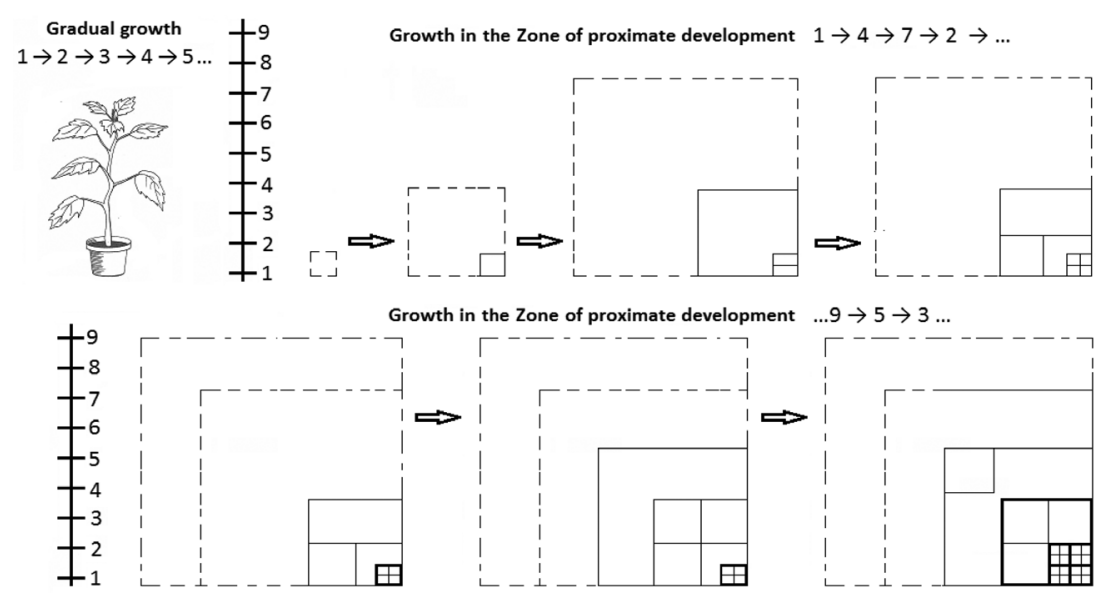

Figure 1: Development of more complex structures is likely not consecutive along the hierarchic levels $(1-2-3 \ldots)$ (size of squares) but is a ZPD-like interaction between the upper- and the lowerlevel (1-4-7-2-...). 
at the level of gross anatomy. In sum, in most consistent structures there is no strict association of $i$-1 level structures with only one $i$-level structure as $i-1$ level structures can contribute to several $i$-structures, recursively between levels (i.e. with an overlapping $i+1$ build-up), and these contributions vary in time.

4. Fundamental to the evolution, or emergence of complex structures is the relative diversity of substructures, in many senses of the word "relative".

Modern evolution theory recognized that the classical portrayal of evolution as a ladder of progress leading towards better traits for adaptation and fitness is insufficient to explain many phenomena in biology. Instead of a drive to perfection, evolution appears to be associated with a drive for diversification (not necessarily with increasing complexity) $[6,7,9,15]$. One of the main benefits of having a diversity of systems at say, the $i$-level, is that when selection tendencies from the $i+1$-level are not compatible with the $i-1$ components in one $i$-level system, there are alternative $i$-level structures. Otherwise, the $i$-level structures would disappear, compromising existence of other prf-s that relied on it.

Emerging structures have a greater chance to exist as something consistent when they are produced in bunches. Even if not capable of being reproduced exactly they may still form a family (with shareable components) and a distribution of similar even if not identical configurations has a chance for better fitness in changing environments. Uniqueness of prf-s also should be noted: Performances are unique in terms of their components, but should be multiple in numbers. Returning to the play analogy, every single performance of a play and all of the steps leading to each performance are unique in terms of cast and crew, theatre, sets, direction, locale and audience. At the same time similar prf-s have more chance to be observable and to be used at many levels than a single or multiple diverse prf-s. Similar performances appear like structures because they lie close to some mean, thus reinforcing it. Diverse performances fail to do so, hence seem less visible, though this doesn't mean that they don't exist. The existence of living systems depends on the consistency of their components which must emerge as a collection of relatively similar and sequentially compatible, but not necessarily identical states.

5. Diagonal subordination and the overlap of inter-level associations (principle 3) and "family production" (principle 4) lead to another important aspect of dievolution: that configurations survive better as ensembles and as contingent cycles of multiple states, rather than as a structure with well-defined and steady properties. A question arises: how many states in such a cycle can an ensemble have?

"Two types as a minimum" comes to mind, and there is likely more than just matter of a minimum number for multiplicity. As noted above, $i$-level systems have overlapping sets of $i$-1-level components and in theory should compete for these components to maintain their own consistent existence. Competition strategies are one of the extensive areas of evolutionary modelling, however, another overlooked aspect of the $i$ - 1 level overlap in $i$-level structures is the reciprocity of rivals that compete for the same $i$ - 1 -level resources. Examples of reciprocal relationships are numerous not only between biological species but also in the neurotransmitter systems: 5-HT vs. NE; GABA vs. glutamate (Glu); prolactin (PRL) vs. Growth Hormone (GH); Neuropeptide Y (NPY) vs. Substance P (SupP); mu- vs. kappa-opioid receptors (MOPr vs. $\mathrm{KOPr}$ ), and these relationships existed long before the human brain or brain cells in general were formed. The anatomy of the nervous system and the co-localization of neurotransmitter receptors in such reciprocal systems likely are not just the product of the top-level selection (in our case the levels of gross anatomy or of the functional tasks of humans and animals) but the product of an interaction between this top level, $i+1$, and the $i-1$ (metabolic/chemical systems) level, leading to the emergence of the structures at the intermediate, $i$-level (such as brain structures). 
The reciprocal relationships within these neurotransmitter pairs, when they suppress each other's release, represent the idea of the basic contingency cycle, essential for the survival of complex systems [8]. Such cycles are commonly described at a horizontal level of emergence by autocatalytic functions or as prey-predator or host-parasite models, however, these cycles have a better chance of survival if they employ not only horizontal but also diagonal subordination as described above. They have available multiple degrees of freedom and multiple systems and states at several levels, due to the overlap in associated elements, and they can survive contingencies that depend on the status of the $i-1$ and the $i+1$ level systems better than single-configuration systems.

6. Dievolution likely employs the phenomena of fractal functionality and "cruise controls" described in the next section. Formally, we can introduce the following three operators working recursively between levels $[i-n, i+n]$ when a perf $i$ emerges: selection $\Lambda_{\mathrm{i}}$, integration []$_{\mathrm{i}}$ and production $\mathrm{O}_{\mathrm{i}}$. Call them Functional Differentiation operators, or FD-3. The $\mathrm{O}_{\mathrm{i}}$ denote perf-s that emerge no matter how transient they are, and which don't necessarily work as a selection factor for the lower levels. Only the $\Lambda_{i}$ denotes subsets or aspects of these perf processes that are compatible for supporting the emergence of the structures. A most simple prf is: $\mathrm{O}_{\mathrm{i}}=\left([]_{i}\left(\Lambda_{\mathrm{i}+1}, \mathrm{O}_{\mathrm{i}-1}\right)\right.$.

One important aspects of these operators is that they transform to each other's type on a diagonal between levels in the following manner: novel integrations []$_{\mathrm{i}}$ at the $i$ level become routine establishments that can be used at the $i+1$ level if supported by contributing factors, along the ZPD dynamics $\left([]_{i} \leftrightarrow \mathrm{O}_{i+n}\right)$ and cruise control creation $\left([]_{\mathrm{i}} \leftrightarrow \mathrm{O}_{\mathrm{i}-\mathrm{n}}\right)$. Since the operator []$_{\mathrm{i}}$ denotes an integration of a prf at the $i$ level based on compatibility of offers at the upper $\left(\Lambda_{i+n}\right)$ and lower $\left(\Lambda_{i-n}\right)$ levels of complexity in contribution to the emerging performance, such compatibility might be affected from both sides of the diagonal, with the $\Lambda_{i+n} \leftrightarrow[]_{i}$ for the selection as a contribution from the upper levels. However, it is often an oversight that performance capacities of the lower level work as a selective factor in a integration $\Lambda_{i-1}\left(\mathrm{O}_{\mathrm{i}-\mathrm{n}}\right) \leftrightarrow[]_{\mathrm{i}}$, and psychological phenomena of "projection through capacities" or "embodiment" are example of such dynamics $[6,10]$.

\section{FUNCTIONAL DIFFERENTIATION (FD) AND FRACTAL FUNCTIONALITY (FF)}

\subsection{Emerging prf-s as recursions on a diagonal}

The various operations that enter into dievolution are not thought of as acting continuously but rather in quazi-discontinuous cycles, $I I, I 2, I 3$ and so on. Since the operators acting in each cycle will vary as a result of the actions of previous cycles, this takes the form of an iterated function system [40]. Such systems generate attracting sets which form persisting dynamical structures akin to speciation. There is a caveat here which stems from the fact that the state spaces upon which these operators act are not, strictly speaking, identical. In fact the phase spaces associated with the different levels are undergoing repeated change, sometimes increasing in dimensionality, sometimes decreasing, with varying partitioning as the upper levels change and along with them their classifying, discriminating and selecting functions, and with varying membership as elements are created and destroyed. Nevertheless if the time frames for the various levels are sufficiently different then it is possible to model this situation at the $\mathrm{i}-1$ level on a fast time scale as an iterated function system and at the $\mathrm{i}$ and $i+1$ levels on much slower time scales. In this way, there may be time for an attractor to emerge at a lower level for a sufficiently long duration so that there is a transient structure capable of triggering actions from the upper levels onto the lower levels, altering the dynamics and state space, and setting 
off a new iterated function system for the next cycle. In this manner, one expects to see a very complex form of recursion and fractal structure.

4.2 Cruise controls facilitate construction of consistent prfs but also carry functional specificity of contributing factors: FF

The production of prfs that are similar to each other and consistent is challenging, considering the emergent and transient nature of all of the components of these prfs. One approach is to develop habits. Modern psychology recognises that with repetition and learning, mammals construct their behaviour using more and more automatic elements. The common view of habits is that they are "bricks", the most consistent, well-learned elements of behaviour, whether we consider physical activity, verbal activity or cognition. Once learned, driving becomes automatic, speech becomes fluent, concept formation faster. Previous experience, similar to epigenetic (morphogenetic) fields, seems to determine future performance: previously learned habits are activated more easily than new behaviour.

The more consistent and more observable prfs use pre-made units that are supported by previous developments ("cruise controls"). Similar to cruise control in a car, such pre-made and pre-tried units of performance are only useful in a steady, predictable environment. In spite of the evolution of systems facilitating the emergence of structures in more and more complex and changeable settings, there are multiple "cruise controls" observed at the various levels of complexity. We define a "cruise control" as an epigenetically based repetition of previously integrated performances (prf-s) that run using compatible inter-level elements $\mathrm{O}_{\mathrm{i}}=[]_{\mathrm{i}-1}\left(\bigwedge_{\mathrm{i}}, \mathrm{O}_{\mathrm{i}-2}\right)$. These prfs are not being oriented to the upper levels as much as new prfs as they have established support at at least three levels of organization. They are known for their reproductive nature, for example, solitons, genes, memes and habits. These units can be denoted as: []$_{\mathrm{i}-1}=\left\{\mathrm{O}_{\mathrm{i}}\left([]_{\mathrm{i}}\left(\wedge_{\mathrm{i}+1}, \mathrm{O}_{\mathrm{i}-1}\right)\right)\right\}_{\mathrm{j}}=\left\{\mathrm{O}_{\mathrm{i}-1} \mathrm{O}_{\mathrm{i}}\right\}_{\mathrm{j}}$. A distribution of the established prf-s, $\mathrm{O}_{\mathrm{i}}$ (more often than a single established $\mathrm{O}_{\mathrm{i}}$ ) becomes a factor that contributes to selection processes at both lower and upper levels $\left\{\mathrm{O}_{\mathrm{i}-\mathrm{n}} \mathrm{O}_{\mathrm{i}}\right\}_{\mathrm{j}} \leftrightarrow \Lambda_{\mathrm{ij}-1},\left\{\mathrm{O}_{\mathrm{i}+1} \mathrm{O}_{\mathrm{i}}\right\}_{\mathrm{j}} \leftrightarrow \Lambda_{\mathrm{ij}+1}$. Cruisecontrol type performances (i.e. those whose emergence was facilitated by the epigenetic history of their previous integrations) by definition carry functional specificity of the multi-level factors that contributed to their consistent emergence.

Multiple reports from biological sciences point to the impact of the properties of functional activities shaped by the environment of biological species, including socio-cultural and intellectual activities of humans. If we allow a recursive dynamics between several levels of organization as described above, then it is reasonable to expect Fractal Functionality $(F F)$ phenomena: this is when biases in features related to the functional specificity of structures on one level emerge in similar biases at other levels. Indeed, we see similarity in the functional differentiation of elements within living systems related to the functions of storage, support of structural stability, motion, transport of lower-level elements within the system, defense, reproduction, metabolic processing, etc. [22, 30]. Functional diversity of elements expanded dramatically with evolution of living systems but even for systems like atoms and molecules we see differentiation in function between elements that are positioned at the boundary of these systems vs. internally positions, the elements with high structural flexibility vs. low, etc.

Space does not permit to provide more complete analysis of functional differentiation, however the three operators with the FD-3 functions of selection, integration and maintenance of performance can be traced most universally within natural systems of various levels of complexity $[22,30]$. An example of the FF related to these three functions is provided in the last part of this Section. 
4.3 Saving outliers, or how to feed the wolves and to save the sheep

If there is a tendency towards automatizing prfs, then eventually prfs should become similar to one another, risking death should a change in the environment challenge their capacities. Here is a tough choice for nature: (1) to excel in the consistency of structures, multiplying extensively in a stable environment but with high odds of dying in an unstable environment, or (2) to sacrifice consistency by allowing deviations from the means, settling for less than perfect, "good enough" prfs but improving survival in uncertain environments. In simulations the tradeoff between sociability and diversity can be seen as a cycle between a totalitarian mode based on high interconnectivity and compatibility between agents and a structural decomposition mode based on the expansion of horizontal diversity $[18,31]$.

Diagonal subordination and contingency cycles are two solutions to the problem of "saving outliers vs. maintaining the mean" that appeared in evolution. Nature partly solved this problem through sex differentiation, which uses gene recombination. Such recombination is one of the examples of diagonal subordination as the parts of DNA molecules (configurations of "cruise controls" at $i-l$ level) are exposed to selection by different environmental conditions $(i+l$ level) in production of the phenotypes $(i$ - level). What is rarely discussed in regards to sex differentiation is that it provides two partitions of a biological species: one that stores beneficial features of the species, i.e. cruise controls, and another that allows the species to experiment with outliers [32, 33].

The Evolutionary Theory of Sex (ETS) that analysed sex differences in the shape of phenotypic distributions described sexual dimorphism as a functional specialization of a species into two partitions, (1) conservational (female sex) and (2) variational (male sex). Trofimova [34] analysed sex differences in communicative and exploratory abilities and mental disabilities from the perspective of the ETS and suggested that male superiority in risk- and sensation seeking, physical abilities, higher rates of psychopathy, dyslexia, autism, higher birth and accidental death rates reflected of the systemic variational function of the male sex. Female superiority in verbal abilities, lawfulness, socialisation, empathy and agreeableness reflected the systemic conservational function of the female sex. Trofimova also suggested that the male sex (variable partition) plays an evolutionary role of pruning of the redundant excesses in a species' bank of beneficial characteristics in spite of resistance from the conservational partition.

Constructive Neutral Evolution (CNE) theory also describes a "two step" origin-fixation process $[13,14]$. Similar to the ETS, it suggests that the first stage of evolution is the origin of mutations (the ETS describes these emerging in males more often than in females) during which selection processes reinforce and fixate beneficial characteristics (by passing these to female genes). The difference between the CNE and Dievolution theory is that the latter treats the origin of performances and their fixation into cruise controls not just as stages but as two simultaneous directions of evolution working on a diagonal across a vertical hierarchy and horizontal diversity of living systems. It is suggested here that prf-s at several lower $(i-n)$ levels, and not just one level, determine the shape of the Zone of Proximate Development (ZPD) (i.e. the properties of the emerging upper levels of organization). Making cruises" down the diagonal and shaping the ZPD up to the diagonal of levels of organization are two closely integrated processes, even though they happen in different time frames.

\section{AN EXAMPLE OF NEUROTRANSMITTER SYSTEMS REGULATING HUMAN TEMPERAMENT}

Neurotransmitter systems (NT) regulating human temperament provide an especially good example of diagonal evolution as clearly the morphology of their projections in the human brain is a product of coevolution of these systems with the specifics of human activities regulated by several upper 
levels of complexity, as well as changing, unpredictable and complex environments [26-29]. The function of the brain NT ( $i-2$ level) is commonly attributed to a transmission of signals, i.e. informational $\left(\bigwedge_{i+1}\right.$-type) functions tuning the integration of a performance ( $i$-level) by an individual ( $i-1$ level) to a best fit between his/her abilities $\left(\left\{\mathrm{O}_{\mathrm{i}-n} \mathrm{O}_{\mathrm{i}-1}\right\}_{\mathrm{j}}\right)$ and environmental demands $\left(\bigwedge_{\mathrm{i}+1}\right)$. The same $\Lambda_{i+1}$-type chemical composites or their close relatives implicated in somatic functions or peripheral (i.e. not brain) nervous system serve a function of behavioural integration of a physical motion ([ $]_{\mathrm{i}}$-type) "(as in acetylcholine, ACh and prolactin PRL) or the maintenance of physical activities $\left(\mathrm{O}_{\mathrm{i}-1}\right.$-type) (like in neuropeptides). Such associations with different functionalities in the same body illustrate the concept of diagonal subordination and overlapping associations.

Let us focus now on the composition of the $\left(\bigwedge_{i+1}\right.$-type $)$ function of NT and look at the functional differentiation between the main NT groups. Many ancient NT regulate the behaviour of modern humans: amino acids (with initial role as body nutrition, $\mathrm{O}_{\mathrm{i}-\mathrm{n}}$ ), neuropeptides (integrating capacities and the needs of the body, []$_{i-n}$ ) and serotonin (5-HT), including its predecessors (with an initial role of a homeostatic regulator which integrates mind and body with the outside world, including initial light sensitivity, $\Lambda_{\mathrm{i}+\mathrm{n}}$ [35]. Azmitia [35] estimates that the 5-HT precursor tryptophan and its metabolites were present in unicellular organisms nearly 3 millions years ago. To make 5-HT from tryptophan, oxygen is needed, and in the earliest geological times the Earth's atmosphere had little oxygen. Azmitia points out that 5-HT emerged specifically in unicellular systems capable of photosynthesis and the cellular production of oxygen.

Recent research shows that the differences in functional roles within each of these three families of brain neurotransmitters can be also classified using the FD-3 operators. Thus, among amino acids such FD-3 functions can be seen in three main groups: Glu $\left(\mathrm{O}_{\mathrm{i}-\mathrm{n}}\right), \mathrm{ACh}\left([]_{\mathrm{i}-\mathrm{n}}\right)$ and GABA $\left(\bigwedge_{\mathrm{i}-\mathrm{n}}\right)$; among hypothalamic neuropeptides: $\mathrm{GH}\left(\mathrm{O}_{\mathrm{i}-\mathrm{n}}\right)$, PRL $\left([]_{\mathrm{i}-\mathrm{n}}\right)$ and $\mathrm{SOM}\left(\Lambda_{\mathrm{i}-\mathrm{n}}\right)$, orexigenic neuropeptides: orexins $\left(\mathrm{O}_{\mathrm{i}-\mathrm{n}}\right)$, SubP $\left([]_{\mathrm{i}-n}\right)$ and $\operatorname{NPY}\left(\Lambda_{\mathrm{i}-\mathrm{n}}\right)$ and neuropeptidic opioid-receptor systems: MOPr $\left(\mathrm{O}_{\mathrm{i}-\mathrm{n}}\right), \operatorname{KOPr}\left([]_{\mathrm{i}-\mathrm{n}}\right)$ and $\mathrm{DOPr}\left(\Lambda_{\mathrm{i}-\mathrm{n}}\right)$, as well as among monoamine neurotransmitters: 5 - $\mathrm{HT}\left(\mathrm{O}_{\mathrm{i}-\mathrm{n}}\right)$, $\operatorname{DA}\left([]_{i-n}\right)$ and $\mathrm{NE}\left(\Lambda_{\mathrm{i}-\mathbf{n}}\right)[36,37]$.

Reciprocal relationships between pairs within the same families of neurotransmitters were noted above in a context of contingency cycles. Interestingly, in spite of the mutual inhibition of each others' release, in many cases one member of such pairs is a "chemical parent" to another member: Glu to GABA, DA to NE, NE to E, PRL to GH, etc. Within each of these pairs the derivatives appear later in evolution from a "parent" and suppress this parent-neurotransmitter's release. This "production of their own rivals" is clearly beneficial for self-regulation of the amount of NT produced within one system and such ability to self-regulate illustrates that evolution likely continues at several levels of organizational complexity in an iterative and simultaneous manner.

Finally, most consistent biologically-based traits (temperament) regulating human behaviour also exhibit FD-3 specialization (shown as 3 columns in Table 1). Four horizontal groups of traits are given in an evolutional progression of regulatory systems. The 1st row relates to the ancient opioid receptor systems that emerged entangled with the immune system [38] and later developed into the regulation of the most recent monoamine neurotransmitters [38, 39]. Recently, it was found that the body is capable of producing endogenous opioids and of changing the density of endogenous ligands, inducing dispositional emotional states. As the activation of these receptors leads to the release of monoamines, these opioid receptor systems likely play an even more important role in emotionality than do monoamines as they amplify a sense of security (MOPr), alertness and chronic anxiety (KOPr) or an initiation of action (DOPr) [39].

The second group of traits relates to the aspects of actions requiring lesser cortical involvement: either pre-made habits and/or orientation to explicitly present reinforcers. Behavioural regulation in known and/or predictable situations uses habits (learned or explicit elements, either physical or cog- 
Table 1: The contribution of neurotransmitter systems in most stable (temperament) traits according to the neurochemical model Functional Ensemble of Temperament (FET) [36, 37], with notations of the FD-3 operators. Note: 5-HT: serotonin; DA: dopamine; NE: noradrenalin; ACh: acetylcholine; Glu: glutamate; GH: Growth Hormone; SOM: Somatostatin; PRL: prolactin; OXY: oxytocin; SubP: Substance P; NPY: Neuropeptide Y; AdrR, KOPr, MOPr, DOPr: adrenergic, kappa-, mu-, delta-opioid receptors.

\begin{tabular}{lccc}
\hline $\begin{array}{l}\text { Functional } \\
\text { aspects: }\end{array}$ & $\begin{array}{c}\text { Behavioral } \\
\text { orientation to types } \\
\text { of rein-forcers: }\end{array}$ & $\begin{array}{c}\text { Dynamical aspects } \\
\text { Preferred speed of } \\
\text { integration of actions: }\end{array}$ & $\begin{array}{c}\text { Energetic aspects: mainte- } \\
\text { nance of pro-longed and/or } \\
\text { intense activities: }\end{array}$ \\
\hline
\end{tabular}

Regulation by the opioid receptors systems, amplification/appraisal of:

orientational, dynamical and energetic aspects

\begin{tabular}{lccc}
\hline Emotional & $\Lambda_{\mathrm{i}}\left([]_{\mathrm{i}}\right)$ & {[]$_{\mathrm{i}}=\left\{\left\{\Lambda_{\mathrm{i}+\mathrm{n}}^{*}, \mathrm{O}_{\mathrm{i}, \mathrm{i}-\mathrm{n}}\right\}\right\}$} & $\mathrm{O}_{\mathrm{i}}\left(\Lambda_{\mathrm{i}-1}\right)$ \\
amplifier & Neuroticism & Impulsivity & Self-confidence \\
& $\mathrm{KOPr} \rightarrow \mathrm{NE}-\mathrm{HPA}$ & $\mathrm{DOPr} \rightarrow(\mathrm{DA}, \mathrm{MOPr})$ & $\mathrm{MOPr} \rightarrow(5-\mathrm{HT}, \mathrm{DA})$ \\
& $\mathrm{KOPr}>\mathrm{MOPr}$ & & $\mathrm{KOPr}<\mathrm{MOPr}, \mathrm{SOM}$ \\
\hline
\end{tabular}

Regulation by neuropeptide and basal ganglia monoamine systems as a tuning between bodies' capacities and explicitly present, more determined degrees of freedom in behavior

\begin{tabular}{|c|c|c|c|}
\hline $\begin{array}{l}\text { Physical-motor } \\
\text { aspects }\end{array}$ & $\begin{array}{c}\Lambda_{\mathrm{i}}=\left\{\mathrm{O}_{\mathrm{i}-1} * \mathrm{O}_{1-\mathrm{n}}\right) \\
\text { Sensation-seeking } \\
\mathrm{NE}+\mathrm{NPY} / \mathrm{SubP}, \mathrm{AdrR}\end{array}$ & $\begin{array}{c}{[[]]_{\mathrm{i}}=[]_{\mathrm{i}+1}\left\{\left\{\mathrm{O}_{\mathrm{i}-\mathrm{n}}\right\}\right\}} \\
\text { Motor Tempo } \\
\text { DA+PRL+GABA/Glu }\end{array}$ & $\begin{array}{c}\mathrm{O}_{\mathrm{i}+1}[[[]]] \\
\text { Endurance } \\
\text { 5-HT+ACh, GH Orexins }\end{array}$ \\
\hline
\end{tabular}

Regulation by cortical monoamine systems as a tuning between developed blocks of explicit actions and implicit, more probabilistic aspects of situations

\begin{tabular}{cccc}
\hline Mental & $\wedge[]_{\mathrm{i}}=\left(\bigwedge_{\mathrm{i}, \mathrm{i}+\mathrm{n}}\left([]_{\mathrm{i}}\left(\mathrm{O}_{\mathrm{i}, \mathrm{i}-1}\right)\right.\right.$, & {[] $\mathrm{O}_{\mathrm{i}}=[]_{\mathrm{i}}\left(\bigwedge_{\mathrm{i}, \mathrm{i}=1, \mathrm{i}+\mathrm{n}}\left(\mathrm{O}_{\mathrm{i}, \mathrm{i}-1}\right)\right)$} & $\mathrm{O} \Lambda_{\mathrm{i}}=\mathrm{O}_{\mathrm{i}}\left(\Lambda_{\mathrm{i}},[]_{\mathrm{i}},\left(\bigwedge_{\mathrm{i}=1} * \mathrm{O}_{\mathrm{i}-1}\right)\right)$ \\
aspects & $\left.\bigwedge_{\mathrm{i}+2, \mathrm{O}}\right)$ & Plasticity vs. & Mental Endurance \\
& $\begin{array}{c}\text { Sensitivity to } \\
\text { probabilities }\end{array}$ & rigidity & (attention) \\
& $\mathrm{NE}+\mathrm{DA}+\mathrm{ACh}$ & $\mathrm{DA}+5-\mathrm{HT}$ & $\mathrm{NE}, \mathrm{ACh}$ \\
& & & \\
\hline
\end{tabular}

Regulation by monoamine and hormone systems tuned to socialization

\begin{tabular}{cccc}
\hline Social-verbal & $\Lambda_{\mathrm{ij}}=\left\{[]_{i j}\left\{\mathrm{O}_{i} * \mathrm{O}_{k}\right\}\right\}$ & {[]$_{i j}=\left\{\left\{\mathrm{O}_{i k} * \mathrm{O}_{j k}\right\}\right\}$} & $\mathrm{O}_{i j}=[[[]]]_{i j}\left\{\mathrm{O}_{i j} * \mathrm{O}_{k j}\right\}_{i, i-l}$ \\
Empathy-autism & Social-verbal Tempo: & Social-verbal Endurance \\
& NE+OXY, VSP & DA+ PRL, OXY & 5-HT+NP, OXY \\
\hline
\end{tabular}

nitive) that serve as "cruise controls" of behaviour, representing recursive prf components "down the diagonal. The traits of the 3rd row use more cortical involvement for processing socio-cultural context and complex probabilistic environment in general. They developed in evolution as a capacity for "internalization of external reinforcers", helping to map probabilities of events and to create anticipatory behavioural dispositions prior to events. Therefore, these traits are involved in the ZPD of the upper part of the diagonal emergence in building performance of single individual. In this sense, the 
3rd row illustrates the ZPD for human analytic capacities, also distinguished for energetic maintenance of sustained attention (mental endurance, $\mathrm{O}_{\mathrm{i}}$ ), integration and shifts in actions under highly changeable and unpredictable conditions (plasticity, []$_{\mathrm{i}}$ ) and abilities to process information about not immediately present, implicit, abstract and probabilistic features or causes of events (Sensitivity to probabilities, $\Lambda_{\mathbf{i}}$ ) $[36,37]$.

In summary, this article briefly described principles of the theory of diagonal evolution (Dievolution) that uses at least three operators that could be linked to the functionality of elements composing complex systems. These three operators act at minimum three different levels of complexity and might lead to Fractal Functionality within the functional differentiation of elements. The Dievolution theory suggests that the evolution and emergence of living phenomena are a result of the processes happening on a diagonal of a vertical hierarchy and horizontal diversity of living systems, and these processes happen in two directions. Processes from the up-down direction of the diagonal emerge as "cruise controls", or elements with the highest stability at the lower level of organization, and processes from the bottom-up direction of the diagonal emerge in the shaping of the Zone of Proximate Development of upper-level systems.

\section{REFERENCES}

[1] Whitehead, A.N., Process and Reality. An Essay in Cosmology. Gifford Lectures Delivered in the University of Edinburgh During the Session 1927-1928, Cambridge University Press: Macmillan, New York, 1929.

[2] Rosen, R., Effective processes and natural law. In The Universal Turing Machine. A HalfCentury Survey, ed. R.Herken, Oxford University Press, pp. 523-537, 1988.

[3] Goldstein, J., Emergence as a construct: history and issues. Emergence: Complexity \& Organization, 1(1), pp. 49-72, 1999. http://dx.doi.org/10.1207/s15327000em0101_4

[4] Trofimova, I., Universals and specifics in psychology. In Nonlinear Dynamics in Life and Social Sciences, eds. W. Sulis \& I. Trofimoval, IOS Press: Amsterdam, pp. 286-307, 2001.

[5] Trofimova, I.N., Who is in charge of science: men view "Time" as more fixed, "Reality" as less real, and "Order" as less ordered. Cognitive Systems Research, 15-16, pp. 50-56, 2012. http://dx.doi.org/10.1016/j.cogsys.2011.07.001

[6] Trofimova, I., Observer bias: how temperament matters in semantic perception of lexical material. PLoS One, 91, p. e85677, doi:10.1371/journal.pone.0085677, 2014. http://dx.doi.org/10.1371/journal.pone.0085677

[7] Cairns-Smith, A., Genetic Takeover, Cambridge University Press, 1982

[8] Gould, S.J., The Structure of Evolutionary Theory, The Belknap Press of Harvard University Press: Cambridge, MA. pp. 365-382, 2002.

[9] Vrba, E. \& Gould, S., The hierarchical expansion of sorting and selection: sorting and selection cannot be equated. Paleobiology, 122, pp. 217-228, 1986.

[10] Trofimova, I., How people of different age, sex and temperament estimate the world. Psychological Report, 85, pp. 533-552, 1999.

http://dx.doi.org/10.2466/PR0.85.6.533-552

[11] Trofimova, I., Understanding misunderstanding: a study of sex differences in meaning attribution. Psychological Research, 776, pp. 748-760, 2012.

http://dx.doi.org/10.1007/s00426-012-0462-8

[12] Dawkins, R., The Blind Watchmaker, Norton \& Company: New York, 1986. 
[13] Stoltzfus, A., On the possibility of constructive neutral evolution. Journal of Molecular Evolution, 49, pp. 169-181, 1999.

http://dx.doi.org/10.1007/PL00006540

[14] Stoltzfus, A., Constructive neutral evolution: exploring evolutionary theory's curious disconnect. Biology Direct, 7, p. 35, 2012.

http://dx.doi.org/10.1186/1745-6150-7-35

[15] Gould, S.J. \& Lewontin, R.C., The spandrels of San Marco and the Panglossian paradigm: a critique of the adaptationist program. Proceeding of the Royal Socirty London B, 205, pp. 581-598, 1979.

http://dx.doi.org/10.1098/rspb.1979.0086

[16] Dawkins, R., Climbing Mount Improbable, Norton \& Company: New York, 1996.

[17] Barkow, J.H., Darwin, Sex, and Status: Biological Approaches to Mind and Culture, University of Toronto Press: Toronto, ON, 1989.

[18] Trofimova, I., Principles, concepts and phenomena of Ensembles with variable structure. In Nonlinear Dynamics in Life and Social Sciences, eds. W. Sulis \& I. Trofimoval, IOS Press: Amsterdam. pp. 217-231, 2001.

[19] Sulis, W., Stochastic phase decoupling in dynamical networks. Nonlinear Dynamics, Psychology, and Life Science, 124, pp. 327-358, 2008.

[20] Wilson, D.S. \& Wilson, E.O., Rethinking the theoretical foundation of sociobiology. Quarterly Review of Biology, 824, pp. 327-348, 2007.

http://dx.doi.org/10.1086/522809

[21] Sulis, W., Collective intelligence: observations and models. In Chaos and Complexity in Psychology, eds. S.J. Guastello, M. Koopmans \& D. Pincus, Cambridge University Press, pp. 41-72, 2009.

[22] Trofimova, I., Functional differentiation in developmental systems. In Unifying Themes in Complex Systems, ed. Y. Bar-Yan, Perseus, pp. 557-567, 2000.

[23] Trofimova, I., Potapov, A. \& Sulis, W., Collective effects on individual behavior: in search of universality. International Journal of Chaos Theory and Applications, 3, pp. 53-63, 1998.

[24] Thompson, J.N., The Coevolutionary Process, University Chicago Press, 1994. http://dx.doi.org/10.7208/chicago/9780226797670.001.0001

[25] Heckman, T.M. \& Kauffmann, G., The coevolution of galaxies and supermassive black holes: a local perspective. Science, 333(6039), pp. 182-185, 2011.

http://dx.doi.org/10.1126/science.1200504

[26] Sober, E. \& Wilson, D.S., Unto Others: The Evolution and Psychology of Unselfish Behavior, Harvard University Press: Cambridge, MA, 1998.

[27] Leontiev, A.N., Problems of the Development of the Mind, Trans. M. Kopylova. Moscow: Progress Publishers, 1981.

[28] Altman, J., Three levels of mentation and the hierarchic organization of the human brain. In Psychology and Biology of Language and Thought, eds. G.A. Miller \& E. Lennerberg, Academic Press: New York, pp. 90-107, 1978.

[29] MacLean, P., The Triune Brain in Evolution, Plenum: New York, 1989.

[30] Trofimova, I.N., Universal principles of evolution as a foundation for analysis of human nature. Philosophy Research, 3, pp. 5-23, 1995 in Russian.

[31] Trofimova, I., Sociability, diversity and compatibility in developing systems: EVS approach. In Formal Descriptions of Developing Systems, eds. J. Nation, I. Trofimova, J.D. Rand \& W. Sulis, Kluwer Press. pp. 231-248, 2003.

http://dx.doi.org/10.1007/978-94-010-0064-2_13 
[32] Geodakyan, V.A., Sexual dimorphism. In Evolution and Morphogenesis, eds. J. Mlikovsky \& V.J. Novak, Academia, Praha, pp. 467-477, 1985.

[33] Geodakyan, S.V., Two Sexes. Why? The Evolutionary Theory of Sex, Wilmington, USA, 2012.

[34] Trofimova, I., Do psychological sex differences reflect evolutionary bi-sexual partitioning? American Journal of Psychology, 1284, pp. 485-514, 2015.

http://dx.doi.org/10.5406/amerjpsyc.128.4.0485

[35] Azmitia, E., Evolution of serotonin: sunlight to suicide. In Handbook of Behavioral Neurobiology of Serotonin, eds. C. Muller \& B. Jacobs, Elsevier: New York, 2010. http://dx.doi.org/10.1016/S1569-7339(10)70069-2

[36] Trofimova, I. \& Robbins, T.W., Temperament and arousal systems: a new synthesis of differential psychology and functional neurochemistry. Neurosciences \& Biobehavioural Reviews, doi: 10.1016/j.neubiorev.2016.03.008, 2016.

[37] Trofimova, I., The interlocking between functional aspects of activities and a neurochemical model of adult temperament. In Temperaments: Individual Differences, Social and Environmental Influences and Impact on Quality of Life, ed. M.C Arnold, Nova Science Publishers, Inc.: New York, pp. 77-147, 2016.

[38] Stefano, G.B. \& Kream, R., Endogenous opiates, opioids, and immune function: evolutionary brokerage of defensive behaviors. Seminars in Cancer Biology, 18, pp. 190-198, 2008. http://dx.doi.org/10.1016/j.semcancer.2007.12.001

[39] Bodnar, R.J., Endogenous opiates and behavior. Peptides, 32, pp. 2522-2552, 2011. http://dx.doi.org/10.1016/j.peptides.2011.09.020

[40] Barnsley, M., Superfractals, Cambridge University Press: Cambridge, 2006. http://dx.doi.org/10.1017/CBO9781107590168 